\title{
Tratamiento periodístico de la prohibición de las corridas de toros en Cataluña en la prensa nacional española desde la perspectiva del framing
}

\author{
Noa María CARballa Rivas \\ Universidad Pontificia de Salamanca \\ nmcarballari@upsa.es \\ Aurora GARCÍA GONZÁLEZ \\ Universidad de Vigo \\ auroragg@uvigo.es
}

Recibido: 1 de agosto de 2013

Aceptado: 16 de mayo de 2014

\section{Resumen}

Con la prohibición de las corridas de toros en el Parlamento de Cataluña, el 28 de julio de 2010, se sucedieron multitud de reacciones, a favor y en contra, y también se creó un debate mediático sobre el supuesto carácter nacionalista e independentista de Cataluña. En este estudio se presta especial atención a los encuadres noticiosos de los diarios nacionales, la prensa editada en Barcelona y los diarios salmantinos, teniendo en cuenta la especial tradición taurina y ganadera de la provincia de Salamanca. De este modo, se pudieron observar grandes diferencias en cuanto a la cobertura informativa.

Palabras clave: framing, prensa, análisis de contenido, Cataluña, corridas de toros

\section{The informative treatment of bullfights in Spanish national press. An analysis of content from the Framing perspective}

\begin{abstract}
With the prohibition of the bullfights in the parliament of Cataluña on july $28^{\text {th }}$ in 2010 , many manifestations happened in favor or against it, and also it was created a debate in the media about the nationalist and in independence character of Cataluña. The focus of this study is in the frames of the three national journals, three journals of Barcelona and three journals of Salamanca focusing the especial tradition of the bullfights and the livestock in Salamanca. Thus, it was observed great differences respecting the informative coverage.

Keywords: framing, press, content analysis, Cataluña, bullfights

\section{Referencia normalizada}

CARBALLA RIVAS, Noa María y GARCÍA GONZÁLEZ, Aurora (2014): “Tratamiento periodístico de la prohibición de las corridas de toros en Cataluña en la prensa nacional española desde la perspectiva del framing". Estudios sobre el Mensaje Periodístico. Vol. 20, Núm. 2 (julio-diciembre), págs.: 969985. Madrid, Servicio de Publicaciones de la Universidad Complutense.
\end{abstract}

Sumario: 1. Introducción. 2. Metodología e hipótesis. 3. Las corridas de toros en España. 4. La prohibición de los toros en Cataluña. 5. Encuadres noticiosos. 6. Resultados. 7. Conclusiones. 8. Referencias bibliográficas

\section{Introducción}

La presente investigación aborda el tratamiento informativo de los distintos periódicos nacionales sobre el debate de la prohibición de las corridas de toros en Cataluña.

Para ello se pretende dar respuesta al siguiente problema: ¿Hay diferencias significativas entre los diversos medios según el contexto en el que se publica? 
Sobre la base de esta cuestión se delimitaron dos objetivos primordiales. En primer lugar, definir cuál fue el tratamiento informativo de la decisión tomada en el Parlamento de Cataluña, con el fin de detectar cuáles son los diferentes encuadres utilizados por la prensa española de ámbito nacional para elaborar su información. El segundo objetivo del estudio fue determinar hasta qué punto es diferente o similar el tratamiento informativo realizado entre la prensa local de Salamanca, los periódicos editados en Barcelona y las cabeceras nacionales.

Para alcanzar estos objetivos se optó por una metodología basada en el análisis de contenido que integrase dos paradigmas: cuantitativo y cualitativo.

Por un lado, a la hora de elaborar las hipótesis y tratar los datos se combinaron el proceso inductivo y el deductivo. Los datos que se han empleado para este artículo provienen de la lectura de las publicaciones relativas al tema de estudio en los periódicos impresos, así como de entrevistas personales realizadas a diferentes actores del debate sobre la prohibición de las corridas de toros en Cataluña (ganaderos, políticos, toreros, activistas, críticos taurinos, etc.). Por otro lado, el análisis de los datos textuales se realizó con la metodología del análisis de contenido que analiza material cualitativo mediante procedimientos estadísticos que se complementan con interpretaciones cualitativas.

Una de las características destacables de esta investigación es el interés y novedad del tema. Hasta la fecha, no se han encontrado artículos científicos sobre el tratamiento informativo que han hecho los medios de comunicación sobre la prohibición de las corridas de toros en Cataluña'. Por lo tanto, a pesar de tratarse de un estudio que emplea metodologías comunes como el análisis de contenido y el análisis de encuadres oframes, el tema que se aborda en este trabajo genera interés, no sólo por su carácter novedoso y polémico, sino también por ser el primero que estudia de manera científica en el ámbito de la comunicación el tratamiento informativo de la prohibición de las corridas de toros en Cataluña.

\section{Metodología e hipótesis}

Este estudio incluye diferentes técnicas de investigación: el análisis de contenido y las entrevistas personales, que ayudaron a contextualizar los hechos.

Como una primera aproximación, el análisis de contenido se puede definir como una técnica científica (objetiva y sistemática), que estudia cuantitativamente material cualitativo no estructurado/libre (Urchaga, 2009). Esta técnica se puede emplear dentro de cualquier tipo de investigación científica, ya se inductiva o deductiva, y en diferentes ámbitos, sin embargo, tradicionalmente se aplica al estudio de mensajes que se dan en la comunicación (Sánchez, 2005).

1 La mayor parte de los documentos que se aproximan al estudio que se aborda en la presente investigación se enmarcan en el ámbito jurídico, como por ejemplo ¿Una iniciativa legislativa popular justificativa?: el debate en el Parlament de Catalunya sobre las corridas de toros, de J. De Lucas o El régimen jurídico de los espectáculos taurinos populares y tradicionales: el carnaval del toro de Ciudad Rodrigo, de D. F. De Gatta. 
El problema de investigación a partir del cual parte este estudio es el siguiente: ¿Hay diferencias significativas entre los diversos medios según el contexto en el que publicaron?

Después de la revisión teórica y de una primera lectura del material recopilado, se plantearon las siguientes hipótesis con la pretensión de su corroboración en este estudio:

Hipótesis 1: El tratamiento informativo de los periódicos analizados va a cambiar en función al carácter evaluativo de los textos. Se espera que los periódicos editados en Barcelona ofrezcan un carácter evaluativo positivo (a favor de la prohibición) y que los diarios no editados en Barcelona contengan un carácter evaluativo negativo (en contra de la prohibición).

Hipótesis 2: Se esperan diferencias según el encuadre:

- H2.1.: El encuadre 'Libertad' predomina en los periódicos nacionales y locales de Salamanca.

- H2.2.: Los encuadres 'Maltrato animal'y 'Derechos de los animales' predominan en los periódicos catalanes.

- H2.3.: El encuadre 'Interés económico y turístico' predomina en los periódicos locales de Salamanca.

- H2.4.: Los encuadres 'Nacionalismo' y 'Política/debate' son predominantes en todas las cabeceras analizadas.

- H2.5.: Esta sub-hipótesis se plantea como exploratoria, por lo que se formula de manera interrogativa: ¿Los encuadres 'Tradición', 'Identidad cultural', 'Ecología' e 'Indemnización económica' se presentan de la misma manera en los diferentes periódicos?

- H2.6.: La prensa nacional española y la prensa local salmantina van a presentar menos diversidad de encuadres temáticos que los diarios editados en Barcelona.

El universo o población de análisis del presente trabajo estuvo compuesto por todas las noticias, reportajes y/o entrevistas, editoriales y cartas al director que fueron publicadas por los periódicos de ámbito nacional, locales de Salamanca y los periódicos editados en Barcelona en los meses de julio y agosto de 2010.

En total, se revisaron nueve cabeceras (El País, El Mundo, ABC, La Vanguardia, El Periódico de Catalunya, Avui, La Gaceta de Salamanca, El Adelanto de Salamanca y Tribuna de Salamanca), un total de 558 periódicos (nueve diarios por día), durante un período de tiempo de 62 días (desde el 1 de julio de 2010 hasta el 31 de agosto de 2010). Este proceso de selección y localización de unidades de análisis permitió identificar y someter a análisis 650 unidades.

A partir de la exploración íntegra de los periódicos seleccionados se extrajeron únicamente aquellas unidades de análisis que hacían referencia explícita a la aprobación de la ley que prohíbe las corridas de toros en la comunidad catalana: sus causas y sus consecuencias. Es decir, el tema central de la información es la prohibición de las corridas de toros en la Comunidad Catalana, quedando excluidas las noticias, entrevistas, reportajes y demás información publicada sobre tauromaquia en la que la prohibición de las corridas de toros en Cataluña no actúa con un rol protagónico.

Las unidades de análisis que compusieron la población seleccionada para estudiar el carácter evaluativo de los textos fueron las distintas publicaciones (noticias, repor- 
tajes, columnas/viñetas, cartas al director...) sobre la prohibición de las corridas de toros en Cataluña, que se divulgaron en los meses de julio y agosto de 2010, recogiendo así las distintas reacciones y manifestaciones. Para estudiar los frames, las unidades de análisis fueron las noticias.

\section{Las corridas de toros en España}

La afición por los toros y los festejos taurinos en la Península Ibérica es muy antigua. El origen de la Fiesta se comprende entre la segunda mitad del siglo XV y finales del siglo XVII. No obstante, resulta difícil precisar la época a la que se remonta la festividad taurina con exactitud. Asimismo, conviene señalar el momento en el que los jinetes abandonan los caballos para enfrentarse al toro de pie, en la arena:

Atribúyesele a Francisco Romero, de Ronda, fundador de toda una dinastía de toreros, el haber sido el primero en matar toros cuerpo a cuerpo a pie firme, esperándolos y hurtándose a su acometida gracias a la muleta, de la cual pasa también por ser el inventor, y se fija el año 1726 como aquel que vio nacer tan arriesgada suerte (Tapia, 1992: 13).

La obra El espectáculo más nacional es una de las primeras que estudia la historia de la Fiesta y reúne por primera vez los datos más antiguos conocidos, a partir del S. XI, referentes a las corridas (López, 1987). Tras esta obra, llegarían más investigaciones acerca de los orígenes de la Fiesta, el interés por la prehistoria española, el estudio de las representaciones de toros rupestres....

Las corridas de toros han inspirado a los mayores artistas y a muchos pensadores. Basta con atender a los escritos de intelectuales y eruditos que han dedicado algunas de sus obras a la tauromaquia. Fernando de Rojas hizo alusión en La Celestina a la fiesta de los toros (Macías, 2000) y Miguel de Cervantes incluyó en Don Quijote de la Mancha célebres frases que se han hecho ya inmortales, como "apártate hombre del diablo, apártate del camino, que te harán pedazos esos toros".

Además de inspirarse en la Fiesta, los artistas también se han iluminado por la belleza del toro, que ha fascinado a muchos, tanto a los que asisten a las plazas como a los que no. "Como dijo Unamuno: aunque aborrezco las corridas, me gustan los toros en el campo, y mucho. Algunos de mis mejores ratos los he pasado en una ganadería del campo de Salamanca, dibujando" (Jiménez, 1981: web).

La Fiesta ha servido de motivo de inspiración a multitud de pintores, escultores, dibujantes... Goya retrató toreros y pintó escenas taurinas. Su creación más importante con respecto a las corridas de toros ha sido la serie de grabados La Tauromaquia. Con esta obra Goya se refugiaba tras las calamidades vividas en la fiesta de los toros, a la que tan aficionado fue en su juventud (Pérez, 1979). Otro ejemplo de creación inspirada en la Fiesta es el de Pablo Picasso, muestra de ello, su obra El Guernica.

En cuanto a la cría de ganado de lidia, la región de Castilla y León representa una de las actividades más características y tradicionales, siendo Salamanca la provincia de producción de toro bravo por excelencia a nivel nacional.

Castilla y León es la segunda región española con mayor número de ganaderías de toro de lidia, después de Andalucía. La Comunidad cuenta con 44.347 animales repartidos en 56.804 hectáreas, de las cuáles 48.184 pertenecen a la provincia de Sala- 
manca (Consejería de Agricultura y Ganadería de la Junta de Castilla y León, 2011: web). Según el Centro Etnográfico y Bibliográfico virtual del Toro de Lidia de la Junta de Castilla y León (2012: web), Salamanca cuenta con 221 ganaderías. Las restantes provincias de la región no suman apenas una décima parte de las que alberga la provincia charra. Aún así, la cabaña brava vallisoletana es la segunda en número de explotaciones en la Comunidad Autónoma, seguida por la de Ávila.

\section{La prohibición de los toros en Cataluña}

Barcelona ha sido uno de los centros taurinos más importantes dentro de la cultura mediterránea. De hecho, fue la única ciudad del mundo que tuvo tres plazas de toros activas: el Torín, en La Barceloneta; Las Arenas y La Monumental. Además, en Gerona se ubica la segunda plaza de toros más antigua de España, la de Olot.

Al hablar de la tradición de la tauromaquia en Cataluña se puede abordar la práctica ganadera en la comunidad catalana, las escuelas de tauromaquia, las plazas de toros y los toreros nacidos y/o formados en Cataluña, pero ante todo la historia de las costumbres taurinas. Cataluña es uno de los lugares en los que se tiene uno de los registros más antiguos de correbous. En Cardona está documentado un correbous en 1409 (Barraycoa, 2011). No obstante, González (1996) indica que la primera corrida de toros celebrada en Barcelona de la que se tiene noticia se realizó en 1387 para Joan I, en la Plaza del Rey y en 1585 se celebra en Lérida la primera corrida documentada.

Desde 1989 una serie de municipios catalanes ${ }^{2}$ se han ido declarando antitaurinos de manera progresiva y, a lo largo de los años, en la comunidad catalana, se han sucedido multitud de manifestaciones contra este tipo de fiestas.

2 Tossa de Mar (1989), Vilamacolum (1991), La Vajol (1991), Palafrugell (1991), Calonge (1997), Barcelona (2004), Torelló (2004), Calldetenes (2004), Olot (2004), Ripoll (2004), Tavertet (2004), Manlleu (2004), Granollers (2004), San Feliu del Llobregat (2004), Valls (2004), Badía del Vallès (2004), Molins de Rei (2004), La Roca (2004), Sitges (2005), Sant Cugat (2005), Bellpuig (2005), Banyoles (2005), Abrera (2005), Cerdanyola (2006), Sant Andreu de la Barca (2006), Mollet del Vallès (2006), Teià (2006), Sant Quirze de Besora (2006), Gironella (2006), Biure de l'Alt Empordà (2006), Cabrera de Mar (2006), Cabanes de l'Alt Empordà (2006), Sant Iscle de Vallalta (2006), Guissona (2006), Moià (2006), Artesa de Segre (2006), Vilabertran (2006), Sanaüja (2006), Torrelavit (2006), Torrebesses (2006), Riudarenes (2006), Fornells de la Selva (2007), Brunyola (2007), La Fatarella (2007), Morera de Montsant (2007), Calella (2007), Dels Pallerrols (2007), La Bisbal (2007), Sant Cebrià de Vallalta (2008), Palamós (2008), Les Franqueses del Vallès (2009), Castellar del Vallés (2009), Tagamanent (2009), Pallejà (2009), Arenys de Munt (2009), Caldes de Montbui (2009), Hostalric (2009), Vacarisses (2009), Santa Eulàlia de Ronçana (2009), Aiguafreda (2009), Sant Pere de Vilamajor (2009), Sabadell (2009), Vilassar de d'Alt (2009), Martorell (2009), Castellbisbal (2009), Vallgorgina (2009), Sentmenat (2009), Sant Cebrià de Vallalta (2008), Palamós (2008), Les Franqueses del Vallès (2009), Castellar del Vallés (2009), Tagamanent (2009), Pallejà (2009), Arenys de Munt (2009), Caldes de Montbui (2009), Hostalric (2009), Vacarisses (2009), Santa Eulàlia de Ronçana (2009), Aiguafreda (Barcelona, Cataluña, 2009), Sant Pere de Vilamajor (2009), Sabadell (2009), Vilassar de d'Alt (2009), Martorell (2009), Castellbisbal (2009), Vallgorgina (2009), Sentmenat (2009), Sant Esteve de Palautordera (2009), Arenys de Mar (2009), Begues (2010). 
Después de varias iniciativas prohibicionistas, en 2008 se presenta como Iniciativa Legislativa Popular (ILP) una Proposición de Ley que prohíbe las corridas de toros y la Mesa del Parlamento de Cataluña la acepta a trámite. El Parlamento de la Comunidad Autónoma de Cataluña aprueba la decisión de abolir las corridas taurinas el 28 de julio de 2010, por 68 votos a favor, 55 en contra y nueve abstenciones. El apoyo mayoritario de CiU y de su líder, Artur Mas, a la propuesta, respaldada también por ERC e ICVEUiA, hizo posible que prosperara esta iniciativa, mientras que el PPC y Ciudadanos votaron en contra, como la mayoría de diputados del grupo del PSC. Se trata de la Ley 28/2010, de 3 de agosto, de modificación del Texto Refundido de la Ley de Protección de los Animales, que prohíbe las corridas de toros, pero no las fiestas con toros sin muerte del animal (correbous). El Boletín Oficial del Estado reflejó lo siguiente:

"La consideración del toro como un ser vivo capaz de sufrir ha arraigado en el sentimiento de la sociedad catalana. Esta perspectiva ya ha sido adoptada anteriormente en países de gran tradición democrática, con el resultado de que en el pasado se prohibieron este tipo de espectáculos. El grado de sensibilización de nuestra sociedad, que se traduce en posiciones favorables a la protección de los animales, el fuerte descenso en Cataluña de la afición a las corridas de toros, el rechazo que producen en muchas de las personas que visitan nuestro país espectáculos que, en parte, se nutren de dinero público propician un paso más, el definitivo, hacia la prohibición de las corridas de toros en todas las modalidades establecidas por el Reglamento estatal de espectáculos taurinos, aprobado por el Real decreto 145/1996, de 2 de febrero (BOE, Ley 28/2010: 73974).

Justamente un año después de que el Parlamento catalán prohibiera las corridas de toros, el Gobierno central reconoce la tauromaquia como una disciplina artística y un producto cultural, considerando que Cultura debería asumir todas las cuestiones relacionadas con la promoción y fomento de esa disciplina artística, los estudios, estadísticas y análisis sobre la materia y también el registro de profesionales del sector (BOE, Real Decreto 1151/2011). De esta manera, las competencias en materia taurina pasan del Ministerio de Interior al Ministerio de Cultura. Asimismo, la Comisión Consultiva Nacional de Asuntos Taurinos pasa también a formar parte de Cultura. Además, el 8 de noviembre de 2011 se da a conocer la creación del Premio Nacional de Tauromaquia, que instituye Ángeles González-Sinde Reig, Ministra de Cultura. Este premio pretende dar reconocimiento de la actividad taurina como disciplina artística a cualquier profesional de la tauromaquia en todas sus diferentes manifestaciones (BOE, Orden CUL/3009/2011).

Cuando el Partido Popular llegó al Gobierno (20 de noviembre de 2011), uno de los objetivos fue recuperar las emisiones de las corridas de toros en la cadena pública. En 2006 se decidió vetar este tipo de contenidos en TVE en horario de protección infantil. De este modo, la última corrida emitida en directo por TVE fue el 14 de octubre de 2006.

Seis años después, en 2012, una audiencia muy parecida vuelve a sintonizar La 1 de TVE para ver la corridas de toros. Concretamente, la Feria de Valladolid, emitida el 6 de septiembre de 2012.

Por otro lado, el mismo recurso que se utilizó para prohibir las corridas de toros en Cataluña, también se empleó desde el mundo taurino para defender su fiesta: una ini- 
ciativa legislativa popular. En este caso serían necesarias más firmas, ya que la ILP para la prohibición de las corridas de toros en Cataluña se llevó a cabo a nivel regional, necesitando 180.000 firmas, mientras que la ILP taurina, de dimensión nacional, necesitaba 500.000 firmas para que se pudiera debatir en las Cámara Baja si las corridas de toros son o no Bien de Interés Cultural.

La recogida de firmas se inició el 15 de marzo del 2011, siendo la fecha límite de devolución el 28 de diciembre de ese mismo año, no obstante, la Mesa de la Diputación Permanente del Congreso de los Diputados prorrogó el plazo hasta el 26 de marzo de 2012.

El 22 de marzo de 2012 se presentaron 605.235 firmas que demandaban una Iniciativa Legislativa Popular (ILP) para declarar los toros Bien de Interés Cultural en toda España, bajo la supervisión de 4.764 fedatarios (EFE, 2012: web). De las firmas recogidas, la Junta Electoral dio por válidas 500.165 firmas, entre las cuales, 168.000 provinieron de Cataluña.

\section{Encuadres noticiosos}

Conceptualmente, se ha adaptado el vocablo 'framing' a una de las teorías que abarca la comunicación. Gran número de investigadores han planteado diferentes definiciones para referirse a los encuadres noticiosos o news frames. Según el Diccionario Collins, el sustantivo 'frame' significa 'estructura abierta que da forma y soporte a algo', 'construcción', 'sistema'o 'marco'. Como verbo, 'elaborar', 'construir'o 'dar forma'. La traducción que han adoptado los teóricos de 'Teoría del framing' es 'Teoría del encuadre', ya que el concepto 'framing' suele ser traducido por 'marco', 'encuadre' o 'enfoque', aunque este último se relaciona más con el campo de la fotografía. No obstante, en función del contexto, se emplea 'frame' o 'framing' si la traducción al español no resulta del todo precisa ${ }^{3}$.

Según Entman (1993), 'framing' es el proceso en el que se seleccionan algunos aspectos de la realidad percibida, haciéndolos más sobresalientes en el texto comunicativo, de tal manera que se consiga promover una definición del problema particular, una interpretación causal, una evaluación moral y/o una recomendación de tratamiento para el asunto descrito. Por lo tanto, el autor plantea una idea de encuadre que contiene varias interpretaciones. Diversos teóricos han aportado definiciones que siguen esta línea. Muñiz (2007) recoge las aportaciones de diversos autores y entre las más recientes está la de Ross, que señala que el framing determina la relevancia de la información y establece un contexto para su comprensión y, a su vez, Nelson, indica que los encuadres son descripciones e interpretaciones de una misma información, problema o solución.

Por otra parte, Igartua et al. (2005) señalan que el encuadre se refiere a la perspectiva o enfoque de una información que se manifiesta en la elección e importancia atribuida a los diferentes elementos relacionados, como puede ser la ubicación geográfica, el espacio temporal o los actores protagonistas.

3 A lo largo del presente estudio se puede observar el uso indiscriminado del sustantivo 'frame' y del gerundio de 'to frame', 'framing', refiriéndose al mismo concepto. 
Además, Canel y Sanders (2006) apuntan igualmente que se trata de contenido manifiesto (que incluye elementos simbólicos) que puede evocar contenido ausente y que, haciendo uso de resonancias culturales, ayudan a hacer más comprensible el mundo social, a la vez que implican un juicio sobre el mismo.

Desde estos y otros aportes sobre el framing, Entman (1993) asegura que el concepto 'encuadre' está muy ligado a la idea de selección, por ello uno de los primeros elementos a tener en cuenta a la hora de considerar los frames es la visibilidad, entendiendo que encuadrar es seleccionar aspectos de la realidad percibida y hacerlos sobresalir, de tal manera que se promueva una interpretación, definición, una evaluación moral del problema y/o una recomendación para su solución. Por otra parte, Mariel afirma que el enmarcamiento mediático implica en todos los casos dos principales operaciones de visibilidad: por un lado, énfasis o prioridad de ciertos elementos de las noticias; y por otro, simplificación y fraccionamiento de la realidad mediante la selección de un hecho con exclusión de otros, y por medio de la limitación del campo semántico (Mariel, 2011). Por lo tanto, este tipo de análisis de enfoque habilita y ofrece diversas opciones para indagar sobre los procesos de mediatización.

El investigador realiza la propia operación de simplificación de la información en la construcción científica de un encuadre mediático. Se opera una nueva selección mediante la determinación de unidades de análisis e indicadores y se produce una nueva analogía o metáfora al nominar el frame así construido (Mariel, 2011: 75).

En vista de la gran variedad de definiciones, Scheufele (2000) indica que es necesario determinar una definición unificada de encuadre para poder establecer una auténtica teoría.

Lo mismo opina Tankard, citado por Muñiz (2007), para quien resulta preciso que se establezca un concepto propio de framing para poder estudiar aspectos como pueden ser los efectos de los encuadres en el público.

En el presente estudio se aborda cómo el caso de la prohibición de las corridas de toros en Cataluña ha abierto una brecha más entre Cataluña y España. Algunos partidos políticos, como Convergència i Unió ( $\mathrm{CiU})$, defienden que dicha prohibición no es una cuestión identitaria ni un enfrentamiento de Cataluña contra España. Sin embargo, durante varias semanas este tema ha sido un fenómeno mediático caracterizado por un debate nacionalista.

A través del framing veremos qué tipo de informaciones llega sobre este hecho a los ciudadanos, qué formatos se han utilizado para enfocar esta cuestión y si ha habido alguna evolución en los enfoques. Se trata de aportar una explicación metodológica de los usos del framing en comunicación aplicando el análisis de contenido en prensa. "Conocer cómo se acercan los medios a la realidad es un primer paso para comprender cómo realizan su trabajo los periodistas y cuáles son sus posibilidades y limitaciones" (Sádaba, 2008: 21).

Los estudios de análisis textual de noticias demuestran que la información que se maneja y se presenta a través de los medios de comunicación es elaborada con determinados news frames (news frames genéricos y news frames específicos) para captar la atención y/o influir en la opinión pública.

La identificación de los distintos tipos de news frames ha sido una de las preocupaciones de los investigadores del proceso de tratamiento informativo. Ala hora de de- 
terminar los encuadres, el investigador realiza un análisis previo en una muestra de las noticias. Los news frames se identifican por la presencia y ausencia de ciertas palabras clave, frases hechas, fuentes de información, etc. y el primer paso consiste en definir el problema principal, la atribución de responsabilidad, la valoración moral y la recomendación de un tratamiento (Entman, 1993).

En la presente investigación se plantearon 10 frames, después de una primera lectura de las noticias que compusieron el estudio: 'Maltrato del animal', 'Derechos del animal', 'Identidad cultural de España', 'Interés económico y turístico', 'Tradición', 'Libertad', 'Nacionalismo', 'Ecología', 'Política/debate' e 'Indemnización'.

\section{Resultados}

En lo referente al carácter evaluativo de los textos estudiados, se realizó un análisis interjueces, con el fin de estimar la fiabilidad. Para ello se contó con la colaboración de tres jueces. Este análisis se realizó sobre una muestra del 13,71\%. Para ello se escogieron aleatoriamente 10 unidades de cada uno de los nueve diarios analizados.

Los jueces valoraron, de forma independiente, en una escala de 1-3 (1, a favor de la prohibición; 2 , neutral y 3 , en contra de la prohibición) cada una de las unidades.

La fiabilidad interjueces se calculó mediante el procedimiento del coeficiente Alfa de Cronbach, obteniéndose un índice de 0,913 , lo cual indica una alta concordancia entre los jueces. Comprobada dicha fiabilidad, estos mismos jueces analizaron el resto de las unidades del estudio.

Como se puede observar en la tabla 1 , los datos permitieron determinar que el carácter negativo (en contra de la prohibición) fue el dominante en el tratamiento la información sobre la prohibición de las corridas de toros en Cataluña $(54,9 \%)$, siendo menor el porcentaje de unidades con un carácter positivo (15,7\%).

Tabla 1. Carácter evaluativo de las unidades de análisis

\begin{tabular}{cccccc}
\hline & & $\begin{array}{c}\text { A favor de la } \\
\text { prohibición }\end{array}$ & $\begin{array}{c}\text { En contra de la } \\
\text { prohibición }\end{array}$ & Neutral & Total \\
\hline Total & $\mathrm{n}$ & 102 & 357 & 191 & 650 \\
\hline Total & $\%$ & $15,7 \%$ & $54,9 \%$ & $29,4 \%$ & $100 \%$ \\
\hline
\end{tabular}

A esta amplia mayoría de unidades que se posicionan en contra de la prohibición de las corridas de toros se le encuentra sentido si se tiene en cuenta que la mayor parte de los periódicos analizados simpatizan con la fiesta de los toros. No es así el caso de los periódicos editados en Barcelona, a los que corresponden la mayor parte de los textos que tienen un enfoque positivo en cuanto a la prohibición.

En la tabla 2 se puede observar que la mayor parte de los textos publicados en los periódicos nacionales tuvieron un carácter evaluativo negativo con respecto a la prohibición de las corridas de toros en Cataluña ( $E l$ Mundo, $63,7 \%$ y $A B C, 77,6 \%$ ), salvo El País, en el que se encontró equidad entre los textos a favor de la prohibición $(33,3 \%)$, en contra de la prohibición $(33,3 \%)$ y de carácter neutral $(33,3 \%)$. Con respecto a los diarios editados en Barcelona, se observaron altos porcentajes a favor de la prohibición (La Vanguardia, 40,6\%; El Periódico de Catalunya, 46,6\% y Avui, $48,4 \%$ ), seguidos de textos con un carácter evaluativo neutral (La Vanguardia, 39,1\%; 
El Periódico de Catalunya, 31\% y Avui, 37,5\%). Sin embargo, las unidades publicadas en los periódicos de Salamanca mostraron una actitud contraria a la prohibición: La Gaceta (86,7\%), El Adelanto (76,4\%) y Tribuna (70,4\%).

\begin{tabular}{|c|c|c|c|c|c|}
\hline \multirow[b]{3}{*}{ Diarios } & \multicolumn{4}{|c|}{ Tabla 2. Publicación-enfoque } & \multirow[b]{3}{*}{ Tota } \\
\hline & \multirow[b]{2}{*}{$\begin{array}{l}\mathbf{N}^{\circ} \\
\%\end{array}$} & \multicolumn{2}{|c|}{ Enfoque } & \multirow[b]{2}{*}{ Neutral } & \\
\hline & & $\begin{array}{c}\text { A favor de la } \\
\text { prohibición }\end{array}$ & $\begin{array}{c}\text { En contra de la } \\
\text { prohibición }\end{array}$ & & \\
\hline \multirow{2}{*}{ El País } & $\mathrm{n}$ & 13 & 13 & 13 & 39 \\
\hline & $\%$ & $33,3 \%$ & $33,3 \%$ & $33,3 \%$ & $100 \%$ \\
\hline \multirow{2}{*}{ El Mundo } & $\mathrm{n}$ & 2 & 93 & 51 & 146 \\
\hline & $\%$ & $1,4 \%$ & $63,7 \%$ & $34,9 \%$ & $100 \%$ \\
\hline \multirow{2}{*}{$A B C$} & $\mathrm{n}$ & 1 & 97 & 27 & 125 \\
\hline & $\%$ & $0,8 \%$ & $77,6 \%$ & $21,6 \%$ & $100 \%$ \\
\hline \multirow{2}{*}{ La Vanguardia } & $\mathrm{n}$ & 26 & 13 & 25 & 64 \\
\hline & $\%$ & $40,6 \%$ & $20,3 \%$ & $39,1 \%$ & $100 \%$ \\
\hline \multirow{2}{*}{ El Periódico } & $\mathrm{n}$ & 27 & 13 & 18 & 58 \\
\hline & $\%$ & $46,6 \%$ & $22,4 \%$ & $31 \%$ & $100 \%$ \\
\hline \multirow{2}{*}{ Avui } & $\mathrm{n}$ & 31 & 9 & 24 & 64 \\
\hline & $\%$ & $48,4 \%$ & $14,1 \%$ & $37,5 \%$ & $100 \%$ \\
\hline \multirow{2}{*}{ La Gaceta } & $\mathrm{n}$ & 0 & 39 & 6 & 45 \\
\hline & $\%$ & $0 \%$ & $86,7 \%$ & $13,3 \%$ & $100 \%$ \\
\hline \multirow{2}{*}{ El Adelanto } & $\mathrm{n}$ & 1 & 42 & 12 & 55 \\
\hline & $\%$ & $1,8 \%$ & $76,4 \%$ & $21,8 \%$ & $100 \%$ \\
\hline \multirow{4}{*}{ Tribuna } & $\mathrm{n}$ & 1 & 38 & 15 & 54 \\
\hline & $\%$ & $1,9 \%$ & $70,4 \%$ & $27,8 \%$ & $100 \%$ \\
\hline & $\mathrm{n}$ & 102 & 357 & 191 & 650 \\
\hline & $\%$ & $15,7 \%$ & $54,9 \%$ & $29,4 \%$ & $100 \%$ \\
\hline
\end{tabular}

Con estos datos se interpretó que los periódicos nacionales mostraron una tendencia contraria a la prohibición, salvo El País, diario en el que se observó más neutralidad. En los periódicos catalanes se mostró una actitud favorable a la decisión tomada por el Parlamento de Cataluña, mientras que los salmantinos mostraron una amplia mayoría de textos en contra de la prohibición. De esta manera, en el análisis de conglomerados (gráfico 1) se observaron dos grandes grupos: por un lado, El País y los tres diarios catalanes, que mostraron, en general, más textos a favor de la prohibición, y por otro lado, los diarios $A B C, E l$ Mundo y los tres periódicos salmantinos. 


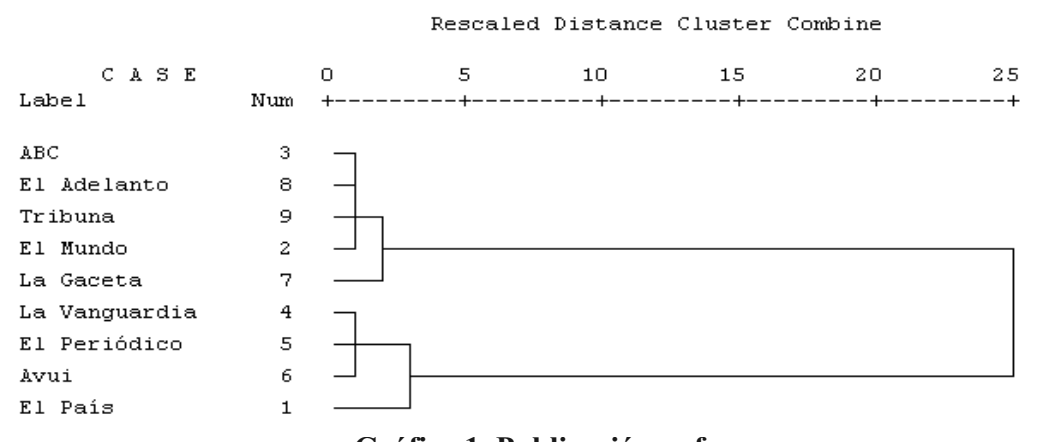

Gráfico 1. Publicación-enfoque

También se estudió la variación del carácter evaluativo de los textos según el género, observando que existían diferencias, en cuanto a que en todos los géneros periodísticos prevaleció un carácter evaluativo en contra de la prohibición (tabla 3). Por un lado, se pudo observar que el género más publicado fue el de opinión (192 columnas/viñetas, 106 cartas al director y 22 editoriales), seguido del meramente informativo (252 noticias). Por otro lado, en todos los géneros se mostró mayoritariamente un enfoque contrario a la prohibición (reportaje, 63,2\%; crónica, 73,7\%; entrevista, $66,7 \%$; editorial, $59,1 \%$; columna/viñeta, $73,4 \%$ y cartas al director, $59,4 \%$ ), salvo en la noticia, en la que se observó un carácter más neutral (57,5\%).

\begin{tabular}{llcccc}
\hline \multicolumn{5}{c}{ Tabla 3. Tabla de contingencia género-enfoque } \\
\cline { 3 - 5 } Géneros & $\mathbf{N}$ & \multicolumn{3}{c}{ Enfoque } & \multirow{2}{*}{ Total } \\
\cline { 3 - 5 } periodístico & $\%$ & $\begin{array}{c}\text { A favor de la } \\
\text { prohibición }\end{array}$ & $\begin{array}{c}\text { En contra de la } \\
\text { prohibición }\end{array}$ & Neutral & \\
\hline \multirow{2}{*}{ Noticia } & $\mathrm{n}$ & 19 & 88 & 145 & 252 \\
& $\%$ & $7,5 \%$ & $34,9 \%$ & $57,5 \%$ & $100 \%$ \\
\hline \multirow{2}{*}{ Reportaje } & $\mathrm{n}$ & 3 & 24 & 11 & 38 \\
& $\%$ & $7,9 \%$ & $63,2 \%$ & $28,9 \%$ & $100 \%$ \\
\hline \multirow{2}{*}{ Crónica } & $\mathrm{n}$ & 0 & 14 & 5 & 19 \\
& $\%$ & $0 \%$ & $73,7 \%$ & $26,3 \%$ & $100 \%$ \\
\hline \multirow{2}{*}{ Entrevista } & $\mathrm{n}$ & 5 & 14 & 2 & 21 \\
& $\%$ & $23,8 \%$ & $66,7 \%$ & $9,5 \%$ & $100 \%$ \\
\hline \multirow{2}{*}{ Editorial } & $\mathrm{n}$ & 6 & 13 & 3 & 22 \\
& $\%$ & $27,3 \%$ & $59,1 \%$ & $13,6 \%$ & $100 \%$ \\
\hline Columna & $\mathrm{n}$ & 31 & 141 & 20 & 192 \\
/Viñeta & $\%$ & $16,1 \%$ & $73,4 \%$ & $10,4 \%$ & $100 \%$ \\
\hline Cartas al & $\mathrm{n}$ & 38 & 63 & 5 & 106 \\
Director & $\%$ & $35,8 \%$ & $59,4 \%$ & $4,7 \%$ & $100 \%$ \\
\hline \multirow{2}{*}{ Total } & $\mathrm{n}$ & 102 & 357 & 191 & 650 \\
& $\%$ & $15,7 \%$ & $54,9 \%$ & $29,4 \%$ & $100 \%$ \\
\hline
\end{tabular}


Por lo tanto, los resultados de nuestro estudio demuestran la verificación de la primera hipótesis, en la que se esperaba que los periódicos editados en Barcelona ofrecieran un carácter evaluativo positivo (a favor de la prohibición) y que los diarios no editados en Barcelona contuviesen un carácter evaluativo negativo (en contra de la prohibición).

Una posible explicación de este hecho podría radicar en la línea editorial de cada periódico y, en el caso particular de los rotativos catalanes, también se encontraría una respuesta sustancial en el Diario Oficial de la Generalitat (DOGC, CLT/1082/2011). En él se hicieron públicas algunas de las subvenciones concedidas por el Gobierno autonómico de Cataluña a los medios de comunicación durante el año 2010, en concreto, más de diez millones de euros a repartir entre las cabeceras catalanas, con el fin de fomentar y consolidar el espacio catalán de comunicación. De hecho, algunos de los medios en soporte papel más beneficiados fueron El Periódico de Catalunya y Avui, con 1.261.404,02 euros y 378.293,62 euros, respectivamente (DOGC, CLT/1082/2011).

En cuanto a los resultados de los encuadres noticiosos, también se pudo comprobar (tablas 4 y 5) que los diarios que más encuadres presentaron fueron $A B C$ y $L a$ Vanguardia (cinco encuadres), seguidos de El Mundo y La Gaceta (cuatro encuadres) y El País, El Adelanto y Tribuna (tres encuadres), resultando Avui con menor número de encuadres (dos tipos de frames).

El diario que más ausencia de encuadres registró fue El País (seis encuadres), seguido de Avui y El Adelanto (cinco encuadres); El Periódico de Catalunya (cuatro encuadres); La Gaceta (tres encuadres) y El Mundo, ABC, La Vanguardia y Tribuna (dos encuadres).

Los datos del estudio también permitieron observar que los encuadres noticiosos más empleados fueron Política/debate y Nacionalismo. Estos frames se mostraron en textos que aludieron a la propia votación de la prohibición de las corridas de toros en el Parlamento de Cataluña, a la libertad de voto por parte de los partidos políticos, a las ponencias expuestas en las jornadas de debate, a la cuestión identitaria y carácter independentista por parte de Cataluña, a la defensa de los correbous y/o a la sentencia del Tribunal Constitucional sobre el Estatut.

En cuanto a la ausencia de encuadres, los frames más ausentes fueron Ecología (sólo aparece en el $A B C$ ), Maltrato (figura en El Mundo, La Vanguardia y El Periódico de Catalunya) y Derechos del animal (se plasma en Tribuna, La Gaceta y La Vanguardia).

Tabla 4. Publicación-encuadres

\begin{tabular}{|c|c|c|c|c|c|c|c|c|c|c|}
\hline & 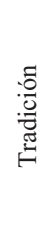 & 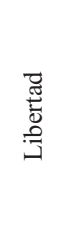 & 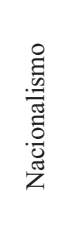 & $\begin{array}{l}\frac{.5}{00} \\
\frac{0}{0} \\
0 \\
0\end{array}$ & 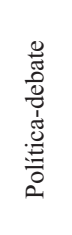 & 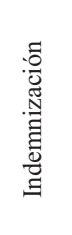 & 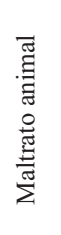 & 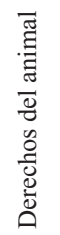 & 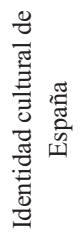 & 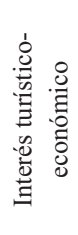 \\
\hline El País & $0 \%$ & $30 \%$ & $60 \%$ & $0 \%$ & $50 \%$ & $10 \%$ & $0 \%$ & $0 \%$ & $0 \%$ & $0 \%$ \\
\hline
\end{tabular}




\begin{tabular}{|l|c|c|c|c|c|c|c|c|c|c|}
\hline El Mundo & $13 \%$ & $27,5 \%$ & $40,6 \%$ & $0 \%$ & $33,3 \%$ & $7,2 \%$ & $2,9 \%$ & $0 \%$ & $30,4 \%$ & $13 \%$ \\
\hline ABC & $22 \%$ & $56,1 \%$ & $36,6 \%$ & $2,4 \%$ & $46,3 \%$ & $2,4 \%$ & $0 \%$ & $0 \%$ & $31,7 \%$ & $17,1 \%$ \\
\hline $\begin{array}{l}\text { La } \\
\text { Vanguardia }\end{array}$ & $20,8 \%$ & $25 \%$ & $54,2 \%$ & $0 \%$ & $75 \%$ & $0 \%$ & $16,7 \%$ & $12,5 \%$ & $25 \%$ & $4,2 \%$ \\
\hline El Periódico & $22,2 \%$ & $0 \%$ & $50 \%$ & $0 \%$ & $50 \%$ & $5,6 \%$ & $11,1 \%$ & $0 \%$ & $0 \%$ & $11,1 \%$ \\
\hline Avui & $0 \%$ & $3,1 \%$ & $56,3 \%$ & $0 \%$ & $62,5 \%$ & $3,1 \%$ & $0 \%$ & $0 \%$ & $0 \%$ & $3,1 \%$ \\
\hline La Gaceta & $7,7 \%$ & $30,8 \%$ & $30,8 \%$ & $0 \%$ & $53,8 \%$ & $0 \%$ & $0 \%$ & $7,7 \%$ & $61,5 \%$ & $15,4 \%$ \\
\hline El Adelanto & $11,8 \%$ & $0 \%$ & $11,8 \%$ & $0 \%$ & $70,6 \%$ & $0 \%$ & $0 \%$ & $0 \%$ & $41,2 \%$ & $23,5 \%$ \\
\hline Tribuna & $4 \%$ & $12 \%$ & $4 \%$ & $0 \%$ & $60 \%$ & $4 \%$ & $0 \%$ & $4 \%$ & $24 \%$ & $20 \%$ \\
\hline \multicolumn{1}{r|}{ Total } & 31 & 59 & 96 & 1 & 128 & 10 & 8 & 5 & 61 & 31 \\
\hline
\end{tabular}

En su mayoría, los diarios no editados en Barcelona presentaron los frames estudiados con un enfoque negativo hacia la prohibición. Por ejemplo, en los siguientes titulares: "La farsa nacionalista acaba con los toros en Cataluña" $(A B C, 29$ de julio de 2010), "...Y ahora a la caza de las muñecas flamencas en las tiendas de recuerdos" ( $A B C, 29$ de julio de 2010), "El catalanismo traduce el no a los toros como un adéu Espanya" (ABC, 29 de julio de 2010) y "Todos ven la sombra nacionalista" (El Mundo, 30 de julio de 2010).

Por el contrario, los periódicos catalanes ofrecieron un enfoque positivo sobre la base de estos frames, sirvan de muestra los siguientes ejemplos: "Els correbous són indispensables en les festes de 26 municipis de l'Ebre" (El Periódico de Catalunya, 25 de julio de 2010), "Adéu Espanya negra" (Avui, 29 de julio de 2010), "L'abolició projecta la singularitat de Catalunya als mitjans internacionals" (El Periódico de Catalunya, 29 de julio de 2010) y "La protecció dels correbous s'ajusta a la legalitat" (Avui, 6 de agosto de 2010).

Con respecto a la cuestión nacionalista, cabe tener presente que durante las semanas previas a la votación, la sentencia del Tribunal Constitucional sobre el Estatut, resuelta el 26 de junio de 2010, supuso numerosas publicaciones en los medios de comunicación, incluida la prensa que se analizó para el presente estudio. Todos los periódicos incluyeron en sus portadas la resolución, así como sus consecuencias, como la manifestación del 10 de julio en Barcelona con el lema "Som una nació, nosaltres decidim" ("Somos una nación, nosotros decidimos"). Además, varias publicaciones recientes que trataron el debate de la prohibición de las corridas de toros en Cataluña relacionaban directamente la sentencia del Estatut con la prohibición de las corridas de toros (Boix, 2011; March, 2011). Por este motivo, se consideró incluir la cuestión de la sentencia del Estatut dentro del encuadre 'Nacionalismo'.

Aunque los temas 'Maltrato animal' y 'Derechos de los animales' fueron los grandes ausentes en la mayor parte de los periódicos, en los diarios catalanes se pudieron observar varios textos que trataron estas cuestiones. La Plataforma PROU! puso sobre la mesa del Parlamento catalán la Iniciativa Legislativa Popular, con el objetivo de velar por los derechos de los animales. Pero los periódicos reflejaron el debate político que se creó durante y después de la votación: los diarios se valieron principalmente de fuentes políticas, que se mostraron en los textos que se publicaron durante 
días (reportajes, entrevistas, etc.). De esta manera, el debate no versó en el maltrato o en los derechos de los animales, sino en la cuestión identitaria y la libertad, como resultó ser en los periódicos nacionales y locales de Salamanca. La mayor parte reflejó en numerosos textos el tema 'Libertad', oponiéndose al hecho de prohibir y reivindicando el derecho de los aficionados a asistir a las corridas de toros. De este modo, los lectores no sólo percibieron los temas sobre los que pensar, sino también la importancia de cada tema, en función a la frecuencia de publicación de estos frames.

Resulta particular el caso de los diarios de Salamanca con respecto al frame 'Ecología'. Habiendo recalcado la importancia de la figura del toro de lidia en la provincia y, en general, en Castilla y León, no se comprende el hecho de que ninguna noticia se basara en este tema. Tal como concibe Entman (1993) el framing -como un proceso de selección de la realidad- los tres periódicos de Salamanca deberían haber tratado esta cuestión. Sobre todo teniendo en cuenta las numerosas listas que se muestran en el marco teórico, elaboradas por diversos autores, en las que se incluyen el interés humano y las consecuencias, que en este caso resultan prácticamente incuestionables (Warren, 1975; De Sousa, 1981; Núñez, 1995; Casals, 2005; Vallvey, 2011). Por lo tanto, la ausencia de este frame pone de manifiesto que, a pesar de que la agenda de la prensa salmantina se centró en la prohibición de las corridas de toros en Cataluña, no se trató un tema de vital importancia para la provincia, como es la preservación de la dehesa y del toro bravo.

\section{Conclusiones}

El trabajo realizado permite establecer al menos las siguientes conclusiones acerca del tratamiento informativo realizado por los diferentes periódicos:

- Existen diferencias notables en el tratamiento periodístico que realizaron los distintos diarios nacionales y esto pone de manifiesto los diferentes intereses de las empresas informativas a la hora de acercar la prohibición de las corridas de toros en Cataluña a los lectores.

- La cobertura del tema de estudio, que formó parte de la agenda informativa de todos los diarios analizados, principalmente de los periódicos El Mundo y $A B C$, varió en función del enfoque empleado por las distintas cabeceras analizadas: los diarios editados en Barcelona mostraron más textos favorables a la prohibición (Avui, 48,4\%; El Periódico de Catalunya, 46,6\% y La Vanguardia, $40,6 \%$ ), mientras que los no editados en Barcelona (los nacionales y salmantinos) reflejaron un enfoque en contra de la prohibición, principalmente $\mathrm{La} G a$ ceta de Salamanca (86,7\%), ABC (77,6\%) y El Mundo $(63,7 \%)$.

- Todos los periódicos analizados incluyeron las noticias de la prohibición de las corridas de toros en Cataluña bajo el paraguas de 'Política/debate' y 'Nacionalismo'. Además, se observaron diferencias en cuanto al tratamiento del frame 'Nacionalismo': por lo general, los diarios editados en Barcelona lo emplearon con un carácter evaluativo positivo, mientras que los no editados en Barcelona, mostraron una tendencia evaluativa negativa.

- Los temas 'Maltrato animal' y 'Derechos de los animales' no tuvieron presencia en la mayor parte de los diarios, pero en la prensa catalana sí se pudieron en- 
contrar textos que presentaron estos frames, principalmente en La Vanguardia ('Maltrato animal', 16,7\%; 'Derechos de los animales', 12,5\%).

- En el caso de la prensa salmantina, los diarios ofrecieron más noticias bajo el encuadre 'Interés económico y turístico' que el resto de diarios analizados. Con este enfoque se pretendía justificar que las corridas de toros generan ingresos económicos y turistas. Sin embargo, La Gaceta de Salamanca, El Adelanto y Tribuna no hicieron referencia alguna a la cuestión ecológica y tampoco aludieron a las características particulares de las dehesas y su ecosistema, ni a la desaparición de la especie en caso de prohibirse las corridas de toros. Esto pone de manifiesto que las empresas informativas de Salamanca se alejaron de los tradicionales criterios de selección de noticias a la hora de publicar contenidos sobre la prohibición de las corridas de toros en Cataluña.

\section{Referencias bibliográficas}

BARRAYCOA, Javier (2011): Historias ocultadas del nacionalismo catalán. Madrid, Libros Libres.

BOE, Ley 28/2010, de 3 de agosto, de modificación del artículo 6 del texto refundido de la Ley de protección de los animales, aprobado por el Decreto legislativo 2/2008.

BOE, Orden CUL/3009/2011, de 3 de noviembre, por la que se modifica la Orden de 22 de junio de 1995, por la que se regulan los Premios Nacionales del Ministerio de Cultura.

BOE, Real Decreto 1151/2011, de 29 de julio, por el que se modifican el Real Decreto 1132/2008, de 4 de julio, por el que se desarrolla la estructura orgánica básica del Ministerio de Cultura y el Real Decreto 1181/2008, de 11 de julio, por el que se modifica y desarrolla la estructura orgánica básica del Ministerio del Interior.

BOIX, Salvador (2011): Toros sí. Una defensa razonada. Madrid, Ediciones Planeta, S.A.

CANEL, María José y SANDERS, Karen (2006): Morality Tales. Political Scandal and Journalism in Britain and Spain in the 1990. New Jersey, Hampton Press.

CASALS CARRO, María Jesús (2005): Periodismo y sentido de la realidad. Teoría y análisis de la narrativa periodística. Madrid, Fragua.

CENTRO ETNOGRÁFICO Y BIBLIOGRÁFICO VIRTUAL DEL TORO DE LIDIA (2012), http://www.cetnotorolidia.es/opencms_wf/opencms/ganaderias/index.html, [Consultado el 13 de mayo de 2013].

CONSEJERÍA DE AGRICULTURA Y GANADERÍA DE LA JUNTA DE CASTILLA Y LEÓN, "Castilla y León apuesta por conservar el patrimonio genético de la raza de lidia", en www.jcyl.es: http://www.jcyl.es/web/jcyl/AgriculturaGanaderia/es/Plantilla100Detalle/1246464862173//1284200338924/Comunicacion?plantillaObligatoria=PlantillaContenidoNoticiaHome, [Consultado el 12 de mayo 2013]. 
DE SOUSA, José Martínez (1981): Diccionario general de periodismo. Madrid, Paraninfo.

DOGC, CLT/1082/2011, de 10 de març, per a qual es dóna publicitat a les subvencions concedides pel Departament de Cultura durant l'any 2010.

EFE (2012): "Los promotores de la ILP taurina anuncian la validación de las 500.000 firmas", en $A B C . e s$, http://www.abc.es/agencias/noticia.asp?noticia=1144721, [Consultado el 13 de mayo de 2013].

ENTMAN, Robert (1993): "Framing: Toward a clarification of a fractured paradigm". Journal of Communication, $\mathrm{n}^{\circ} 43$ (3), pp. 51-58.

GONZÁLEZ, Antoni (1996): Bous, Toros i Braus. Una tauromàquia catalana. Tarragona, Edicions El Mèdol.

IGARTUA, Juan José et al. (2005): "La inmigración en la prensa española. Aportaciones empíricas y metodológicas desde la teoría del encuadre noticioso". Migraciones, $\mathrm{n}^{\circ} 17$, pp. 143-181.

JIMÉNEZ, Salvador (1981): "Escribir de toros", $A B C$, en: http:/hemeroteca.abc.es/nav/Navigate.exe/hemeroteca/madrid/abc/1981/05/16/056 .html [Consultado el 13 de junio de 2013].

LÓPEZ, Juan Gualberto (1987): El espectáculo más nacional. Madrid, Coculsa.

MACÍAS, Raúl (2000): El signo de la Fiesta. Madrid, Egartorre.

MARCH, Paco (2011): Crónicas para un adiós. O no. [2007-2011]. Barcelona, Edicions Bellaterra.

MARIEL, Patricia (2011): “Acción política y frame analysis: convergencias y divergencias entre media frame y marcos de acción colectiva". Perspectivas de la Comunicación, $\mathrm{n}^{\mathrm{o}} 4$ (1), pp. 92-105.

MUÑIZ, Carlos (2007): Encuadres noticiosos e inmigración: del análisis de los contenidos al estudio de los efectos mediáticos, (Tesis doctoral). Salamanca, Universidad de Salamanca.

NúÑEZ, Luis (1995): Introducción al periodismo escrito. Barcelona, Ariel.

PÉREZ, Alfonso (1979): Goya. Caprichos, Desastres, Tauromaquia y Disparates. Madrid, Fundación Juan March.

SÁDABA, Teresa (2008): Framing: el encuadre de las noticias. El binomio terrorismo-medios. Buenos Aires, La Crujía Ediciones.

SÁNCHEZ, José Javier (2005): "Análisis de contenido cuantitativo de medios", en SCHEUFELE, Dietram (2000): "Agenda-setting, priming, and framing revisited. Another look at cognitive effects of political Communications". Mass Communication \& Society, n ${ }^{\circ}$ 3, pp. 297-316.

TAPIA, Daniel (1992): Historia del toreo (1). De Pedro Romero a Manolete. Madrid, Alianza Editorial. 
URCHAGA, José David (2009): “Análisis de Contenido”, en REYES, Román: Diccionario Crítico de Ciencias Sociales, Vol. I. Madrid-México, Universidad Complutense \& Plaza-Valdés, pp. 147-151.

VALLVEY, Fernando Martínez (2011): Teoría del periodismo. Madrid, Centro de Estudios Financieros. Universidad a distancia de Madrid (UDIMA).

WARREN, Carl (1975): Géneros periodísticos. Barcelona, ATE. 